\title{
Sex- and Sports-Specific Epidemiology of Traumatic Lumbar Spine Injuries Sustained During Sporting Activities: Male Snowboarders and Female Horseback Riders at Greatest Risk
}

\author{
Ryan Cheng, B.A., Joseph B. Kahan, M.D., M.P.H., Don Li, Ph.D., \\ Christopher A. Schneble, M.D., and Elizabeth C. Gardner, M.D.
}

Purpose: To generate national estimates of sports-related traumatic lumbar spine injury incidence rates using the National Electronic Injury Surveillance System (NEISS) database and determine any sports- and sex-specific epidemiologic patterns of these traumatic injuries. Methods: Data regarding traumatic lumbar spine injuries sustained through sporting activities from 2009 to 2018 were extracted from the NEISS, a database generating nationwide estimates through patient information collected during emergency department visits from 100 NEISS hospitals across the United States. The estimated number of injuries was calculated using weights assigned by the NEISS database depending on the geographic location of the injury. Results: A total of 497 traumatic lumbar spine injuries were identified over the course of 10 years, suggesting 19,208 estimated injuries. The estimated average injury rate was 6.1 injuries per million persons per year. When analyzed by sex, the incidence rate of traumatic lumbar spine injury was 3.6 injuries per million persons per year for male patients and 2.5 injuries per million persons per year for female patients $(P=.663)$. In male patients, $47 \%$ of the injuries occurred in individuals aged between 10 and 29 years, whereas injuries in female patients were more equally distributed across different age groups. Horseback riding $(27 \%)$, skiing $(5 \%)$, and roller skating $(4 \%)$ were the leading causes of traumatic lumbar injuries in female patients. In male patients, most injuries were experienced as a result of snowboarding (13\%), weightlifting (10\%), and football (6\%). Conclusions: From 2009 to 2018 , the estimated incidence of sports-associated traumatic lumbar spine injuries was approximately 6.1 injuries per million persons per year. Male patients experienced a greater number of traumatic lumbar injuries (3.60) than female patients (2.46), with a relative incidence rate of 1.46. The most common source of injury was snowboarding in male patients and horseback riding in female patients. Level of Evidence: Level III, retrospective comparative study.

$\mathbf{L}$ ow-back pain is one of the most common complaints of athletes at all levels of competition, with some estimates that $30 \%$ of athletes experience an episode of acute low-back pain due to sports. ${ }^{1-4}$ Lumbar spine injuries associated with athletic activities range from minor muscle sprains to catastrophic vertebral fractures with spinal cord injuries. ${ }^{5}$ The ability to appropriately differentiate between benign muscle

From the Department of Orthopaedics and Rehabilitation, Yale School of Medicine, New Haven, Connecticut, U.S.A. (R.C., J.B.K., D.L., C.A.S., E.C.G.); and Department of Cell Biology, Yale School of Medicine, New Haven, Connecticut, U.S.A. (D.L.).

The authors report the following potential conflicts of interest or sources of funding: D.L. received an F30 grant from the National Institute of Diabetes and Digestive and Kidney Diseases, outside the submitted work. Full ICMJE author disclosure forms are available for this article online, as supplementary material.

Received June 26, 2020; accepted December 1, 2020. strains and more severe injuries is critical to determine treatment, which can range from rest and bracing to significant surgery and rehabilitation. The results of this study are valuable for increasing awareness of these injuries in high-risk sports, such as horseback riding and snowboarding, given that sprains and more serious injuries are frequently not well differentiated at first glance.

Address correspondence to Elizabeth C. Gardner, M.D., Department of Orthopedics and Rehabilitation, Yale University School of Medicine, $47 \mathrm{Col}$ lege Ave, New Haven, CT 06511,U.S.A.E-mail:Elizabeth.Gardner@yale.edu Published by Elsevier Inc. on behalf of the Arthroscopy Association of North America. This is an open access article under the CC BY-NC-ND license (http://creativecommons.org/licenses/by-nc-nd/4.0/).

2666-061X/20888

https://doi.org/10.1016/j.asmr.2020.12.001 
Prior studies have shown that sports involving twisting, jumping, axial loading, contact, and repetitive hyperextension have been associated with higher risks of injury. ${ }^{6}$ A 2006 study by Dunn et al. ${ }^{4}$ observed a total of 4,790 college athletes and found that back injuries accounted for $7 \%$ of the total injuries sustained, most of which were reported in football players or gymnasts. Other studies have shown a high incidence of lumbar spine injuries in other sports such as horseback riding, ice hockey, skiing, and snowboarding. ${ }^{7}$

Most research involving lumbar spine injuries in sports is from college or professional databases involving high-level athletes, participating mostly in team sports. The purpose of this study was to generate national estimates of sports-related traumatic lumbar spine injury incidence rates using the National Electronic Injury Surveillance System (NEISS) database and determine any sports- and sex-specific epidemiologic patterns of these traumatic injuries. We hypothesized that these estimates would show a difference in the incidence of lumbar spine injuries across different sports.

\section{Methods}

\section{Data Source}

The U.S. Consumer Product Safety Commission operates the NEISS database, which is accessible through the NEISS Estimates Query Builder. ${ }^{8}$ The NEISS is a stratified sample based on emergency department geographic location and size, in which department size is categorized based on the number of annual emergency department visits reported by each hospital. This probability sample, comprising both community and academic hospitals in the United States and its territories that have at least 6 beds and an emergency department, allows for the analysis of patient information obtained from a nationally representative sample of emergency department visits to 100 NEISS hospitals, as well as the calculation of national estimates from statistical weights assigned to each hospital. For each visit, information regarding the patient's demographic characteristics, location of injury, suspected injury diagnosis, disposition, and activities and products associated with the injury, as well as the narrative, were logged. No specific institutional review board approval was required for this study because the database contains only deidentified patient data.

\section{Patient Selection}

Patient encounters were initially selected using the following search criteria to be included in the sample: lower-trunk injuries; injuries associated with sports or recreational equipment; and diagnostic codes for "crushing," "dislocation," "fracture," and "nerve damage." Although a large number of cases were identified,
Table 1. Incidence Rates Stratified by Sex and Age

\begin{tabular}{ll}
\hline & $\begin{array}{c}\text { Mean Incidence Rate } \\
\text { per Million Persons per Year (Range) }\end{array}$ \\
\hline $\begin{array}{l}\text { Total } \\
\text { Sex }\end{array}$ & $6.05(3.53-8.57)$ \\
$\quad$ Male & $3.60(1.98-5.22)$ \\
Female & $2.46(0.37-4.54)$ \\
Age & \\
$0-9 \mathrm{yr}$ & $0.01(-29.09$ to 29.11$)$ \\
$10-19 \mathrm{yr}$ & $6.04(1.28-10.80)$ \\
$20-29 \mathrm{yr}$ & $4.90(-0.57$ to 10.37$)$ \\
$30-39 \mathrm{yr}$ & $3.62(-3.47$ to 10.71$)$ \\
$40-49 \mathrm{yr}$ & $2.83(-5.40$ to 11.05$)$ \\
$50-59 \mathrm{yr}$ & $1.95(-5.42$ to 9.32$)$ \\
$60-69 \mathrm{yr}$ & $1.20(-7.26$ to 9.66$)$ \\
$\geq 70 \mathrm{yr}$ & $0.70(-7.95$ to 9.35$)$ \\
\hline
\end{tabular}

not all of these cases resulted from participation in sports. Further filtering eliminated incidences involving golf carts, seesaws, teeterboards, diving boards, snow removal tools, portable food or beverage coolers, gocarts, beach chairs, book bags, camping equipment, dune buggies, waterslides, and hammocks. Because not all patients who were admitted to the emergency department had traumatic injuries to the lumbar spine, only narratives containing any of these specific phrases were included for the final data set: L1, L2, L3, L4, L5, lumbar fracture, lumbar $\mathrm{fx}$, transverse process fracture, $\mathrm{TP} \mathrm{fx}$, paralysis, burst fracture, disk herniation, compression fracture, comp $\mathrm{fx}$, L-spine, $\mathrm{fx}$, or disc bulge. Cases pertaining to traumatic injuries of the "lower trunk" or "spine" that did not specify a lumbarrelated injury were not included. Moreover, cases describing a sprain were not included for analysis.

\section{Statistical Analysis}

To calculate the estimated incidence rates per million persons per year, population data were extracted from the U.S. Census Bureau for the period of 2009-2018. Pair-wise comparisons of continuous variables were performed using a 2-tailed unpaired $t$ test of the means. Categorical variables were tested using the $\chi^{2}$ test of contingency tables. Proportions were compared using the $z$ score for multiple comparisons of population proportions. All tests were performed using Stata (version 13; StataCorp, College Station, TX) or Microsoft Excel (Redmond, WA) using a default $\alpha$ level of .05. Results were plotted in Excel, and tables were created in PowerPoint (Microsoft).

\section{Results}

From 2009 to 2018, 497 patients sustained traumatic lumbar spine injuries through sporting activities and presented to NEISS-affiliated hospitals. Of these 497 individuals, 292 were male patients $(59 \%)$ and 205 were female patients $(41 \%)$. Using the weights assigned by the NEISS database depending on the geographic 


\section{DISTRIBUTION OF LUMBAR SPINE INJURIES BY AGE AND SEX}

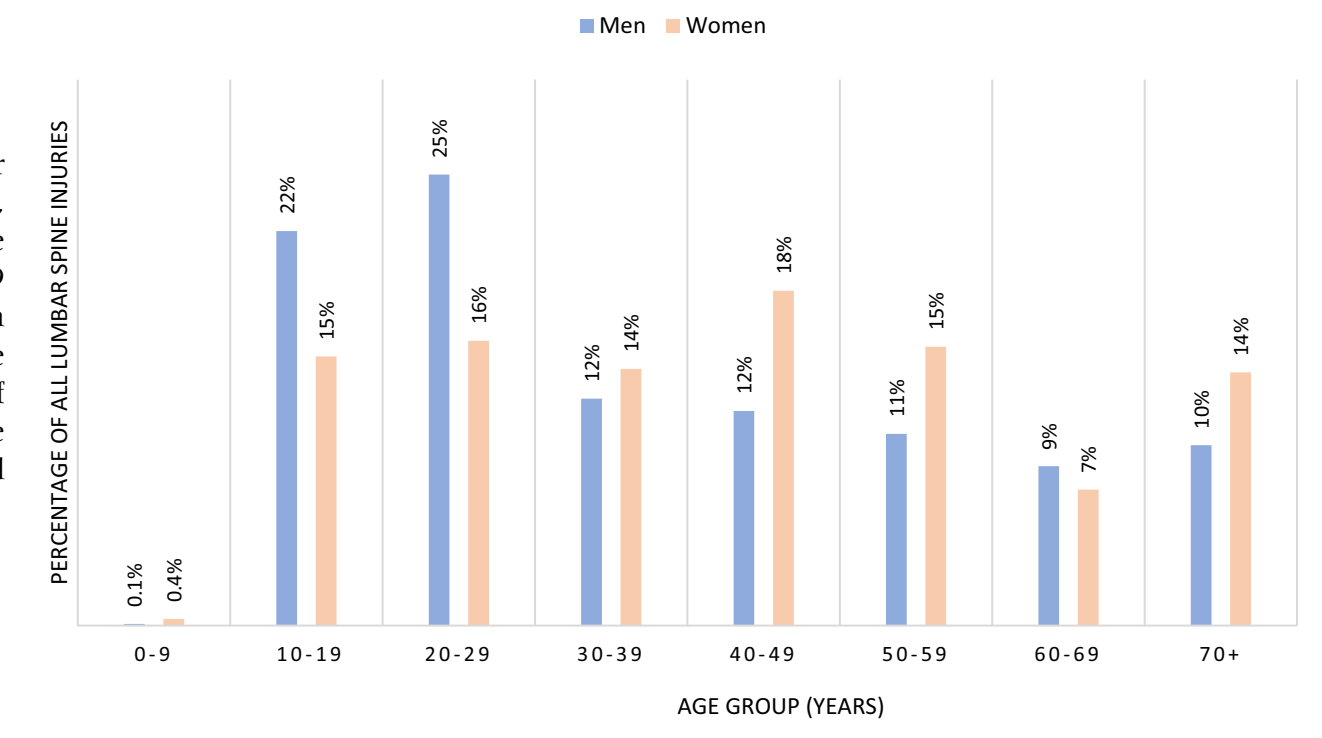

Fig 1. Distribution of lumbar spine injuries across age groups, stratified by sex. Whereas male patients aged between 10 and 29 years were more likely to sustain a lumbar spine injury than the other age groups, the incidence of lumbar spine injuries in female patients was more evenly spread across the age groups.

location of the injury, we determined that this sample represents an estimated 19,208 traumatic lumbar injuries nationally over the 10-year period: 11,414 injuries in male patients and 7,794 in female patients.

The estimated average incidence per million persons per year was 6.05 (95\% confidence interval, 3.538.57). Further incidence rates per million persons per year are shown in Table 1, stratified by both age and sex. Male patients experienced a greater number of traumatic lumbar injuries (3.60) than female patients (2.46) during this period, with a relative incidence rate of 1.46. However, because of the wide confidence intervals, this finding did not reach statistical significance $(P=.663)$. Further analysis of injuries stratified by age and sex is presented in Figure 1, showing increased rates of injuries in male patients among the younger age groups, as well as increased rates of injuries in female patients among the older age groups.

Figure 1 shows the distribution of lumbar spine injuries across age groups, stratified by sex. Male patients aged 10 to 29 years were more likely to sustain a lumbar spine injury than the other age groups. The incidence of lumbar spine injuries in female patients was more evenly spread across the age groups.

During the 10-year period studied, the 5 most frequent sports-associated causes of traumatic lumbar spine injuries, based on annual weighted estimates, were horseback riding (255.1), snowboarding (164.3), weightlifting (148.9), skiing (140.0), and football (63.6) (Fig 2). The incidence of traumatic lumbar sports injuries was significantly associated with the sport played $(P<.001)$.

The weighted estimates of traumatic lumbar injuries were further stratified by sport and sex (Fig 3). For female patients, the highest number of lumbar injuries each year was experienced during horseback riding (estimated 211.9 injuries), followed by skiing (estimated 41.8 injuries) and roller skating (estimated 27.1 injuries). In contrast, the leading causes of injuries in male patients each year were snowboarding (estimated 127.4 injuries), weightlifting (estimated 113.8 injuries), and football (estimated 63.6 injuries). Among all sports, horseback riding accounted for $17 \%$ of total lumbar spine injuries, which was significantly higher than the rates for other sports $(P<.001)$. Among horseback riders, the injuries were heavily concentrated in the 41 to 65 -year-old age group, which accounted for $56 \%$ of the total number of injuries within the sport. Overall, this specific demographic, horseback riders aged between 41 and 65 years, was the most likely to have a traumatic lumbar sports-related injury in our data set $(P=.005)$.

\section{Discussion}

In this study, significant differences in traumatic lumbar spine injuries were seen when patients were stratified by sex and sport. However, lumbar spine injuries can encompass a wide range of conditions from muscle strains to herniated discs, fractures, and spinal cord injuries. ${ }^{5}$ Despite a high prevalence of low-back pain in athletes, ${ }^{1-4}$ there is limited literature regarding national estimates of traumatic lumbar injuries in sports. Our study showed that the rate of traumatic lumbar spine injuries is, fortunately, low (6.05 per million persons per year).

The national incidence of traumatic lumbar spine injuries can be helpful to medical professionals when evaluating and managing athletes with acute low-back 


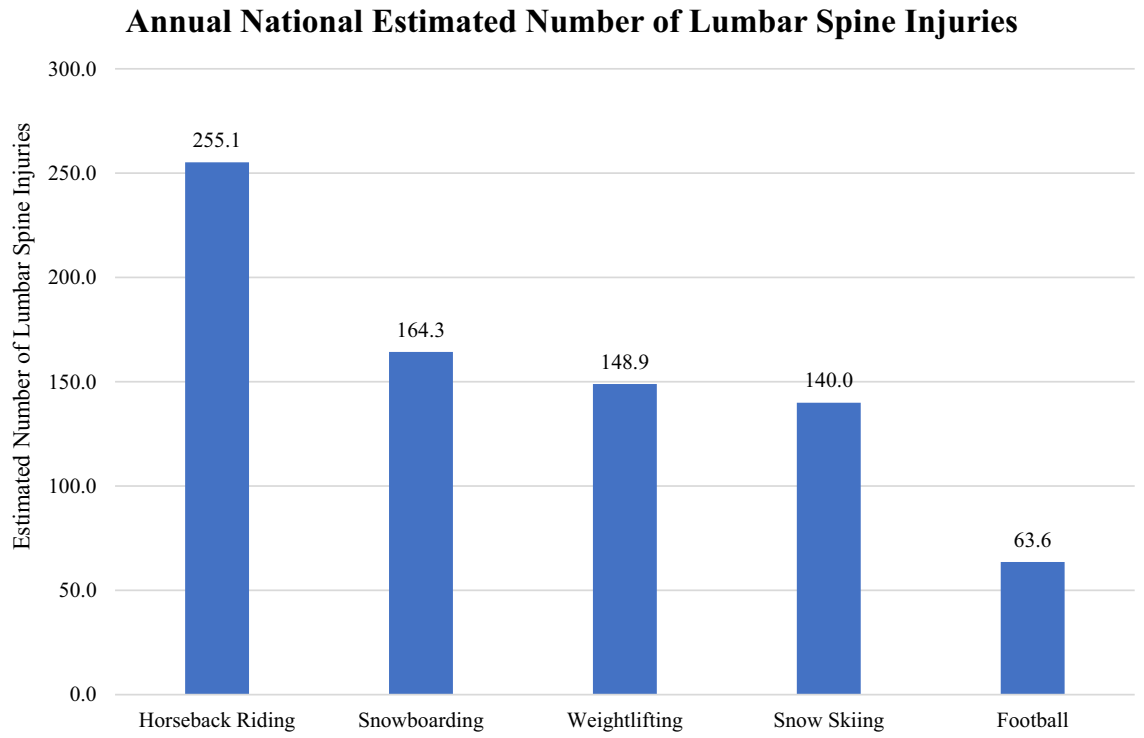

Fig 2. Estimated annual number of lumbar spine injuries associated with each sport. Horseback riding was the sport most commonly associated with a traumatic lumbar spine injury, with 255.1 estimated injuries per year. pain. Although the incidence of traumatic lumbar spine injuries is low, these athletes should be evaluated with caution and a low threshold for further workup in a hospital. Additionally, this information is valuable when developing injury-prevention measures, such as sporting rules and protective equipment.
Prior studies have described the incidence of cervical neck injuries associated with sporting activities ${ }^{10}$; however, no studies have investigated these findings in the lumbar spine. Using the same NEISS database, DePasse et al. ${ }^{10}$ found an overall incidence of 7.74 cervical spine injuries per million person-years,

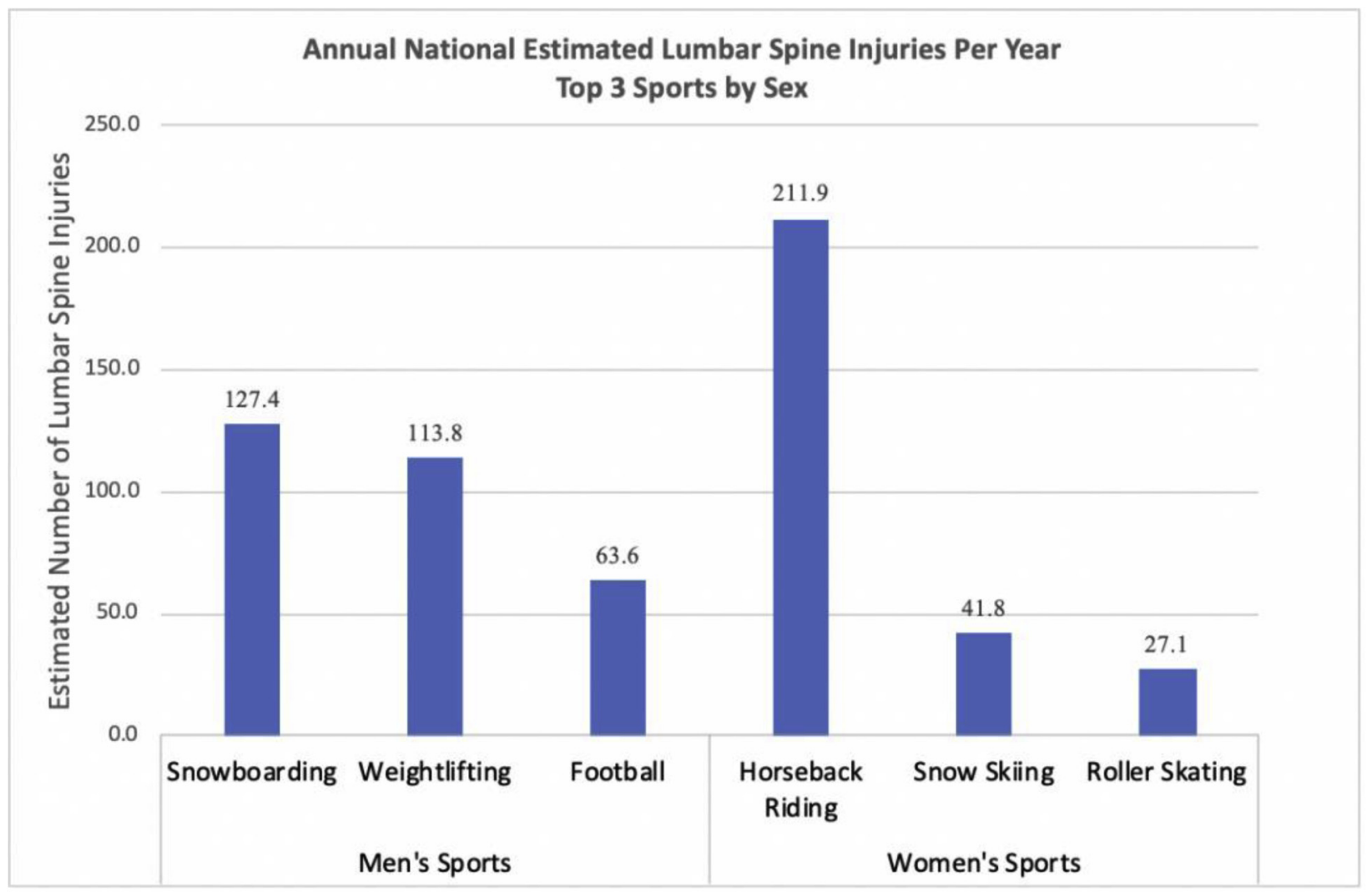

Fig 3. National annual estimates of lumbar spine injuries stratified by sport and sex. The most common sources of injury were snowboarding in male patients and horseback riding in female patients. 
showing similar incidences of cervical and lumbar spine injuries during sporting events. Cervical spine injuries were more common in men who were cycling and women who were horseback riding. Although cycling was not a major cause of lumbar spine injuries, we found a similar high incidence of lumbar spine injuries sustained in female patients while horseback riding.

Our study shows that the demographic most likely to sustain a traumatic lumbar spine injury was horseback riders aged between 41 and 65 years. There are multiple articles describing the injury risks associated with horseback riding, particularly highlighting spinal cord injuries. ${ }^{11,12}$ Lin et al. ${ }^{11}$ conducted a 35-year review of spinal cord injuries sustained during horseback riding and found that horseback riding led to a significantly higher number of injuries in female patients compared with diving, motorcycle riding, and gymnastics. Moreover, a study using the National Spinal Cord Injury Statistical Center database reported that approximately half of all spinal cord injuries in female patients were sustained through horseback riding. ${ }^{13}$ It is interesting to note that the injury profile of horseback riding is different from many other athletic pursuits. A 2002 review article of spinal injuries in horseback riding concluded that there are more lumbar than cervical injuries in horseback riding, in contrast to most other sporting activities. ${ }^{12}$ A comparison between our data and those of DePasse et al. ${ }^{10}$ reinforces this observation, given that an estimated 255.1 annual lumbar spine injuries were sustained in female patients while horseback riding compared with roughly 125 annual cervical spine fractures in the same group. Horseback riders in particular are at increased risk of traumatic lumbar spine injuries, given the falls from a height associated with mounting, jumping, and being thrown off the horse. Overall, horseback riding carries a significant risk of spine injury and a unique risk profile, which our findings support.

In our study, among male patients participating in sporting events, snowboarding, weightlifting, and football were the leading causes of traumatic lumbar spine injuries. Snowboarding has been shown in other studies to have a high incidence of traumatic lumbar spine injuries, ${ }^{1,14,15}$ which our findings support. In a study on snowboard-related spinal injuries conducted by Ball et al., ${ }^{1}$ an incidence rate of 5.73 per 100,000 visits was identified for snowboarders, with lumbar spine injuries accounting for $69.4 \%$ of these injuries. It is thought that in snowboarding, these traumatic lumbar spine injuries are most often due to falls leading to a large axial force on the spine; these more often occur due to falling from a large height after attempting large aerial jumps.

The different leading causes of injuries in male and female patients can potentially be attributed to participation rates in difference sports by male patients versus female patients. Snowboarding, which emerged in the late 1960 s and early 1970s in North America, was pioneered predominantly by men. ${ }^{16}$ Meanwhile, horseback riding has shifted from a male-dominated sport to one in which female equestrians represent over $80 \%$ of the individuals devoted to the field. ${ }^{17}$ As expected, our study showed that the difference in participation rates is reflected within the incidence rates of traumatic lumbar spine injuries sustained by each sex in the respective sports.

Our study found that the highest-risk sports for sustaining a traumatic lumbar spine injury were recreational sports. During recreational sports, in contrast to organized athletics, trained medical professionals are often not immediately available to evaluate or manage injuries, including traumatic lumbar spine injuries. Thus, strategies to educate athletes about protective equipment and injury-prevention measures, particularly in these higher-risk sports, are imperative. A previous study of horseback riders noted that those who were wearing protective jackets at the time of the injury experienced different fracture patterns than those who were not wearing protective equipment. ${ }^{18}$ More specifically, riders who wore protective jackets, often referred to as "body protectors," experienced significantly fewer thoracic spine and lumbar spine injuries than their unprotected counterparts because these protectors serve to cover most of a rider's torso and are designed to limit the amount of impact taken to the rib cage and vital organs during a fall. Although the study had a rather small sample size, the findings suggest the ability to affect injury rates with appropriate equipment.

In snowboarding, the use of back protectors has increased. ${ }^{19}$ However, because there are currently no testing standards specific to snowboarding, the currently available equipment is fashioned based on similar safety equipment for motorcyclists. Ultimately, activity-specific standards should be developed to best protect participants and to minimize any unintended effects of the equipment. Future work will be required to determine whether protective equipment can affect injury rates in snowboarding and other sports.

\section{Limitations}

This study is not without limitations. Using a large database has inherent bias. The NEISS data set creates the possibility of a sampling bias, despite being strategically geographically selected to generate national estimates as accurately as possible. Because national estimates were calculated using weighted numbers assigned to each hospital, small inaccuracies in these weighted values can produce significant errors in estimated incidence rates. Additionally, the use of a database relies on specific types of coders to look through hospital charts and then translate and code the data, thus inviting the possibility of coding and data entry errors, which could lead to inaccurate comparisons 
between sports, age groups, and other categories. It is unknown whether there is a reporting bias regarding organized and recreational sports. There is potential for increased referrals and reports from trainers of organized sports groups that are seeking more informative medical information. However, it is possible that recreational athletes, who do not have access to trainers, are more likely to go to the emergency department than athletes who have more immediate access to medical professionals.

\section{Conclusions}

From 2009 to 2018, the estimated incidence of sportsassociated traumatic lumbar spine injuries was approximately 6.1 injuries per million persons per year. Male patients experienced a greater number of traumatic lumbar injuries (3.60) than female patients (2.46), with a relative incidence rate of 1.46 . The most common source of injury was snowboarding in male patients and horseback riding in female patients.

\section{References}

1. Ball JR, Harris CB, Lee J, Vives MJ. Lumbar spine injuries in sports: Review of the literature and current treatment recommendations. Sports Med Open 2019;5:26.

2. De Luigi AJ. Low back pain in the adolescent athlete. Phys Med Rehabil Clin N Am 2014;25:763-788.

3. Dreisinger TE, Nelson B. Management of back pain in athletes. Sports Med 1996;21:313-320.

4. Dunn IF, Proctor MR, Day AL. Lumbar spine injuries in athletes. Neurosurg Focus 2006;21:E4.

5. Mall NA, Buchowski J, Zebala L, Brophy RH, Wright RW, Matava MJ. Spine and axial skeleton injuries in the National Football League. Am J Sports Med 2012;40: 1755-1761.

6. Lawrence JP, Greene HS, Grauer JN. Back pain in athletes. J Am Acad Orthop Surg 2006;14:726-735.

7. Chan CW, Eng JJ, Tator CH, Krassioukov A, Spinal Cord Injury Research Evidence Team. Epidemiology of sportrelated spinal cord injuries: A systematic review. J Spinal Cord Med 2016;39:255-264.
8. United States Consumer Product Safety Commission. National Electronic Injury Surveillance System (NEISS) estimates query builder, https://cpsc.gov/cgibin/ NEISSQuery/Home.aspx. Accessed November 21, 2020.

9. U.S. and world population clock, https://www.census. gov/popclock/. Published 2019. Accessed November 21, 2020.

10. DePasse JM, Durand W, Palumbo MA, Daniels AH. Sexand sport-specific epidemiology of cervical spine injuries sustained during sporting activities. World Neurosurg 2019;122:e540-e545.

11. Lin CY, Wright J, Bushnik T, Shem K. Traumatic spinal cord injuries in horseback riding: A 35-year review. Am J Sports Med 2011;39:2441-2446.

12. Silver JR. Spinal injuries resulting from horse riding accidents. Spinal Cord 2002;40:264-271.

13. National Spinal Cord Injury Statistical Center. The Spinal Cord Injury Model Systems' data collection syllabus for the National Spinal Cord Injury Database: 2006-2011 project period. https://Www.nscisc.uab.edu/PublicDocuments/ data_collection_syllabus/Syllabus\%202006-2011\%20Revised \%20B-E\%209-10.pdf. Accessed November 21, 2020.

14. Yamakawa H, Murase S, Sakai H, et al. Spinal injuries in snowboarders: Risk of jumping as an integral part of snowboarding. J Trauma 2001;50:1101-1105.

15. Baranto A, Hellstrom M, Cederlund CG, Nyman R, Sward L. Back pain and MRI changes in the thoracolumbar spine of top athletes in four different sports: A 15-year follow-up study. Knee Surg Sports Traumatol Arthrosc 2009;17:1125-1134.

16. Thorpe H. Jibbing the gender order: Females in the snowboarding culture. Sport in Society 2005;8(1):76-100.

17. Adelman M, Knijnik J. Gender and equestrian sport: Riding around the World. New York: Springer, 2013.

18. Kelly PM, Mulhall KJ, Higgins T, et al. The role of back protectors in preventing spinal injuries in horses-riding accidents. Orthop Proc 2018;86-B (suppl II), https:// online.boneandjoint.org.uk/doi/abs/10.1302/0301-620X. 86BSUPP_II.0860122b. Accessed November 21, 2020.

19. Schmitt KU, Liechti B, Michel FI, Stampfli R, Bruhwiler PA. Are current back protectors suitable to prevent spinal injury in recreational snowboarders? Br J Sports Med 2010;44:822-826. 